Fixed Point Theory, 21(2020), No. 2, 657-684

DOI: $10.24193 /$ fpt-ro.2020.2.46

http://www.math.ubbcluj.ro/ nodeacj/sfptcj.html

\title{
NONLOCAL SOLUTIONS AND CONTROLLABILITY OF SCHRÖDINGER EVOLUTION EQUATION
}

\author{
LUISA MALAGUTI* AND KENTAROU YOSHII** \\ *University of Modena and Reggio Emilia, Italy \\ E-mail: luisa.malaguti@unimore.it \\ ** Tokyo University of Science, Japan \\ E-mail: ket.yoshii@gmail.com
}

\begin{abstract}
The paper deals with semilinear evolution equations in complex Hilbert spaces. Nonlocal associated Cauchy problems are studied and the existence and uniqueness of classical solutions is proved. The controllability is investigated too and the topological structure of the controllable set discussed. The results are applied to nonlinear Schrödinger evolution equations with time dependent potential. Several examples of nonlocal conditions are proposed. The evolution system associated to the linear part is not compact and the theory developed in Okazawa-Yoshii [21] for its study is used. The proofs involve the Schauder-Tychonoff fixed point theorem and no strong compactness is assumed on the nonlinear part.
\end{abstract}

Key Words and Phrases: Schrödinger equation, potential with singularities, existence and uniqueness of $\mathrm{C}^{1}$-solutions, nonlocal conditions, controllability, fixed point theorems.

2010 Mathematics Subject Classification: 35Q41, 34B10, 35D35, 93B05, 47H10.

Acknowledgment. The first author is member of the Gruppo Nazionale per l'Analisi Matematica, la Probabilità e le loro Applicazioni (GNAMPA) of the Istituto Nazionale di Alta Matematica (INdAM) and acknowledges financial support from this institution. The paper was prepared during the visit of the second author at the University of Modena and Reggio Emilia.

\section{REFERENCES}

[1] P. Acquistapace, B. Terreni, Linear parabolic equations in Banach spaces with variable domains but constant interpolation spaces, Ann. Scuola Norm. Sup. Pisa Cl. Sci., 13(1986), 75-107.

[2] I. Benedetti, L. Malaguti, V. Taddei, Nonlocal semilinear evolution equations without strong compactness: theory and applications, Boundary Value Prob., 60(2013), 1-18.

[3] I. Benedetti, V. Obukhovskii, V. Taddei, Controllability for systems governed by semilinear evolution inclusions without compactness, Nonlinear Differ. Equ. Appl., 21(2014), 795-812.

[4] I. Benedetti, V. Taddei, M. Väth, Evolution problems with nonlinear nonlocal boundary conditions, J. Dyn. Diff. Equat., 25(2013), 477-503.

[5] S. Bochner, A.E. Taylor, Linear functionals on certain space of abstractly-valued functions, Ann. of Math., 39(1938), 913-944.

[6] H. Brezis, Functional Analysis, Sobolev Spaces and Partial Differential Equations, Springer, New York, 2011. 
[7] T. Chambrion, P. Mason, M. Sigalotti, U. Boscain, Controllability of the discrete-spectrum Schrödinger equation driven by an external field, Ann. Inst. H. Poincaré Anal. Non Linéaire, 26(2009), 329-349.

[8] J. Diestel, W. Ruess, W. Schachermayer, Weak compactness in $L^{1}(\mu, X)$, Proc. Amer. Math. Soc., 118(1993), 447-453.

[9] N. Dunford, J.T. Schwartz, Linear Operators, Wiley, New York, 1988.

[10] P. Hartman, On boundary value problems for systems of ordinary, nonlinear, second order differential equations, Trans. Amer. Math. Soc., 96(1960), 493-509.

[11] M.I. Kamenskii, V. Obukhovskii, P. Zecca, Condensing Multivalued Maps and Semilinear Differential Inclusions in Banach Space, W. de Gruyter, Berlin, 2001.

[12] L.V. Kantorovich, G.P. Akilov, Functional Analysis, Pergamon, Oxford, 1982.

[13] T. Kato, Perturbation Theory for Linear Operators, Grundlehren Math. Wiss., vol. 132, Springer-Verlag, Berlin, New York, 1966; 2nd ed., 1976.

[14] T. Kato, Linear evolution equations of "hyperbolic" type, J. Fac. Sci. Univ. Tokyo, Sec. I., 17(1970), 241-258.

[15] T. Kato, Quasi-Linear Equations of Evolution, with Applications to Partial Differential Equations, Lecture Notes in Math., vol. 448, Springer-Verlag, Berlin, New York, 1975, 25-70.

[16] T. Kato, Abstract Differential Equations and Nonlinear Mixed Problems, Lezioni Fermiane [Fermi Lectures], Accad. Naz. Lincei, Scuola Normale Superiore, Pisa, 1985.

[17] J. Mawhin, Boundary value problems for nonlinear second order vector differential equations, $J$ . Differential Equations, 16(1974), 257-269.

[18] H. Neidhardt, Moving potentials and the completeness of wave operators. III. Existence and completeness, Integral Equations Operator Theory, 15(1992), 100-123.

[19] V. Obukhovskii, P. Zecca Controllability for systems governed by semilinear differential inclusions in a Banach space with anon-compact semigroup, Nonlinear Anal., 70(2009), 3424-3436.

[20] N. Okazawa, Remarks on linear evolution equations of hyperbolic type in Hilbert space, Adv. Math. Sci. Appl., 8(1998), 399-423.

[21] N. Okazawa, K. Yoshii, Linear Schrödinger evolution equations with moving Coulomb singularities, J. Differential Eq., 254(2013), 2964-2999.

[22] A. Paicu, I.I. Vrabie, A class of nonlinear evolution equations subjected to nonlocal initial conditions Nonlinear Anal., 72(2010), 4091-4100.

[23] N. Papageorgiou, Existence of solutions for boundary value problems of semilinear evolution inclusions Indian J. Pure Appl. Math., 23(1992), 477-488.

[24] A. Pazy, Semigroups of Linear Operators and Applications to Partial Differential Equations, Springer-Verlag, Berlin, 1983.

[25] A. Sarychev, Controllability of the cubic Schroedinger equation via a low-dimensional source term, Math. Control Relat. Fields, 2(2012), 247-270.

[26] K. Schmitt, R.C. Thompson, Boundary value problems for infinite systems of second-order differential equations, J . Differential Eq., 18(1975), 277-295.

[27] H. Tanabe, Functional Analytic Methods for Partial Differential Equations, Monographs and Textbooks in Pure and Applied Mathematics, 204. Marcel Dekker, Inc., New York, 1997.

[28] I.I. Vrabie, $C_{0}$-Semigroups and Applications, North-Holland Mathematics Studies, 191, NorthHolland Publishing Co., Amsterdam, 2003.

[29] K. Yajima, On time dependent Schrödinger equations, Dispersive Nonlinear Problems in Mathematical Physics, 267-329, Quaderni di Matematica 15, Dept. Math., Seconda Univ. Napoli, Caserta, 2004.

[30] K. Yoshii, Abstract approach to Dirac equation with time dependent potential, RIMS KôKyûroku, 1892(2014), 101-111.

Received: April 14, 2018; Accepted: January 10, 2019. 